\title{
Tratamento Cirúrgico da Rotura do Septo Interventricular pós Infarto Agudo do Miocárdio
}

Luiz Fernando Leite TANAJURA*, Leopoldo S. PIEGAS*, Luiz Alberto MATTOS*, Ibraim Francisco PINTO*, Hélio M. de MAGALHÃES*, Jaime da Cunha BEMBOM**, Antoninho S. ARNONI*, João Bosco de OLIVEIRA**, Camilo ABDULMASSIH NETO*, Jarbas J. DINKHUYSEN*, Luiz Carlos Bento de SOUZA*, Paulo P. PAULISTA* $^{\star}$, Adib D. JATENE ${ }^{\star \star \star}$, José Eduardo M. R. SOUSA*

TANAJURA, L. F. L.; PIEGAS, L. S.; MATTOS, L. A.; PINTO, I. F.; MAGALHÃES, H. M.; BEMBOM, J. C.; ARNONI, A. S.; OLIVEIRA, J. B.; ABDULMASSIH NETO, C.; DINKHUYSEN, J. J.; SOUZA, L. C. B.; PAULISTA, P. P.; JATENE, A. D.; SOUSA, J. E. M. R. - Tratamento cirúrgico da rotura do septo interventricular pós infarto agudo do miocárdio. Rev. Bras. Cir. Cardiovasc., 2(2): 87-92, 1987.

RESUMO: A rotura do septo interventricular (RSI) reduz a perspectiva de sobrevida do paciente com infarto agudo do miocárdio (IAM). Entre 1968 e 1987, atendemos 48 pacientes (p) com este diagnóstico, sendo $16(33 \%)$ mantidos clinicamente e $32(67 \%)$ submetidos a cirurgia. Todos os p clínicos faleceram durante a internação. Dentre os p operados, o IAM se localizava na parede anterior em $18(56 \%)$ e na inferior em $14(44 \%)$, com mortalidades respectivas de $6(33 \%)$ e $4(29 \%)$. Disfunçāo ventricular esquerda severa (Killip III e IV) foi encontrada em $26(81 \%)$. Em $18(56 \%)$ p foi introduzido o catéter de Swan-Ganz. Dos $31(97 \%)$ p que se submeteram a cinecoronariografia, $22(71 \%)$ apresentavam lesão uniarterial e $9(29 \%)$, doença multiarterial. Entre os p com lesōes isoladas, a descendente anterior foi acometida em $15(68 \%)$, a coronária direita em $6(27 \%)$ e a circunflexa em $1(5 \%)$. A cirurgia foi realizada nas 2 primeiras semanas de evolução em $8(25 \%)$ e, após este período, em $24(75 \%)$, com mortalidades de $6(75 \%)$ e $4(17 \%)$, respectivamente. O balâo intra-aórtico foi usado em $7(22 \%)$. Os procedimentos cirúrgicos associados ao fechamento da RSI foram revascularizaçāo miocárdica em $10(31 \%)$, aneurismectomia de VE em $17(53 \%)$ e infartectomia e $6(19 \%)$. Na evolução tardia, dos 22 sobreviventes, $14(64 \%)$ encontram-se assintomáticos, $1(5 \%)$ sintomático, ocorreram $4(18 \%)$ óbitos e perdeu-se a evolução de $3(13 \%)$. Concluimos que, apesar do risco cirúrgico, a cirurgia precoce ainda é a melhor opção terapêutica desta complicação.

DESCRITORES: septo interventricular, rotura, cirurgia; infarto do miocárdio, cirurgia.

\section{INTRODUÇĀO}

Das complicaçōes do infarto agudo do miocárdio (IAM), a rotura do septo interventricular (RSI) é uma das que apresenta pior prognóstico, cursando com elevada mortalidade. Relatada, inicialmente, por LATHAM ${ }^{10}$ e reconhecida clinicamente, pela primeira vez, por
BRUNN ${ }^{\top}$ teve seu diagnóstico sistematizado por SAGER $^{21}$, em 1934.

Estudos necroscópicos situam sua incidência entre $1,3 \%$ e $2,2 \%{ }^{7}, 16$. VLODAVER \& EDWARDS ${ }^{23}$, estudando 90 casos de roturas ventriculares conseqüentes a infartos do miocárdio, encontraram $20 \%$ de casos com

Trabalho realizado no Instituto Dante Pazzanese de Cardiologia, Sâo Paulo, SP, Brasil.

Apresentado ao 14: Congresso Nacional de Cirurgia Cardiaca. Salvador, BA, 27 e 28 de março, 1987

- Do Instituto Dante Pazzanese de Cardiologia.

*. Do Serviço de Clinica e Cirurgia Cardiovascular Prof. Dr. Adib Jatene. Hospital da Beneficência Portuguesa

*.. Do Hospital do Coraçăo da Associaçăo do Sanatório Sírio.

Endereço para separatas: Luiz Fernando Tanajura. Caixa Postal 215.

01000, São Paulo, SP, Brasil. 
TANAJURA. L. F. L. PIEGAS. L. S: MATTOS. L. A. PINTO. I F: MAGALHAES. H. M: BEMBOM. J C. ARNONI. A S OLIVEIRA. J. B. ABDULMASSIH NETO. C.: DINKHUYSEN. J J SOUZA. L C. B PAULISTA. P P. JATENE. A D.: SOUSA J. E. M. R. - Tratamento cirurgico da rotura do septo interventricular pos infarto agudo do miocardio. Rev. Bras Cir Cardiovasc, 2(2) $87-92.1987$

RSI, percentual inferior às roturas de músculo papilar $(22 \%)$ e das paredes livre do ventrículo esquerdo $(58 \%)$.

O mau prognóstico dos pacientes submetidos apenas a tratamento clinico está bem documentado na literatura. SANDERS et alii ${ }^{22}$ relatam mortalidade de $54 \%$ na primeira semana e $89 \%$ ao fim de 2 meses, cifra idêntica à encontrada por LEE et alii ". OYAMADA \& QUEEN* relatam mortalidades de $87 \%$ e $95 \%$, no 2 . e 12: meses de evolução, respectivamente.

Desde a primeira cirurgia corretiva, realizada por COOLEY et alii ${ }^{4}$, em 1957, vários autores passaram a preconizar o tratamento cirúrgico como o único meio efetivo de controle destes casos ${ }^{18}$. Relatos de literatura situam a mortalidade cirúrgica hospitalar entre $22 \%$ e $65 \%$, sendo esta ampla variação dependente da época em que é realizada a operação ${ }^{2,19}$. Cirurgias realizadas tardiamente teriam mortalidade menor, pois, após o primeiro mês de evolução do IAM, geralmente existe uma boa cicatrização da área infartada, com fibrose das bordas do orifício, o que facilita a manipulaçāo cirúrgica e reduz a possibilidade de recidiva da complicação.

Entretanto, devido ao grave distúrbio hemodinâmico provocado pela RSI, elevado número de pacientes ão consegue alcançar as condiçōes ideais para o tratamento cirúrgico, indo a óbito precocemente ${ }^{12,18}$.

O objetivo deste trabalho é relatar a experiência do Instituto Dante Pazzanese de Cardiologia (IDPC) no manuseio de um grupo de portadores de RSI pós IAM submetidos a cirurgia corretiva.

\section{MATERIAL E RESULTADOS}

Entre 1968 e 1987, foram internados, no IDPC, 48 pacientes com diagnóstico RSI pós IAM, sendo $32(67 \%)$ submetidos a cirurgia e $16(33 \%)$ mantidos clinicamente. Correspondem a cerca de $2,5 \%$ das internaçōes por IAM encontradas neste periodo. Os dois grupos, conforme mostra o Quadro 1, não apresentavam diferença expressiva quanto a sexo, idade, fatores de risco, grau de insuficiência cardiaca, pressões de artéria pulmonar e presença de doença multiarterial.

Apesar de receberem tratamento farmacológico intensivo, inclusive com emprego de contrapulsação com balão intra-aórtico (BIA) em $9(56 \%)$, todos os pacientes clínicos faleceram durante a internaçāo, dos quais 13 $(81 \%)$ nas 2 primeiras semanas de evolução e $3(19 \%)$ após este período.

Dentre os pacientes operados, $25(78 \%)$ eram do sexo masculino e 7 (22\%) do sexo feminino, com idades

\section{QUADRO 1}

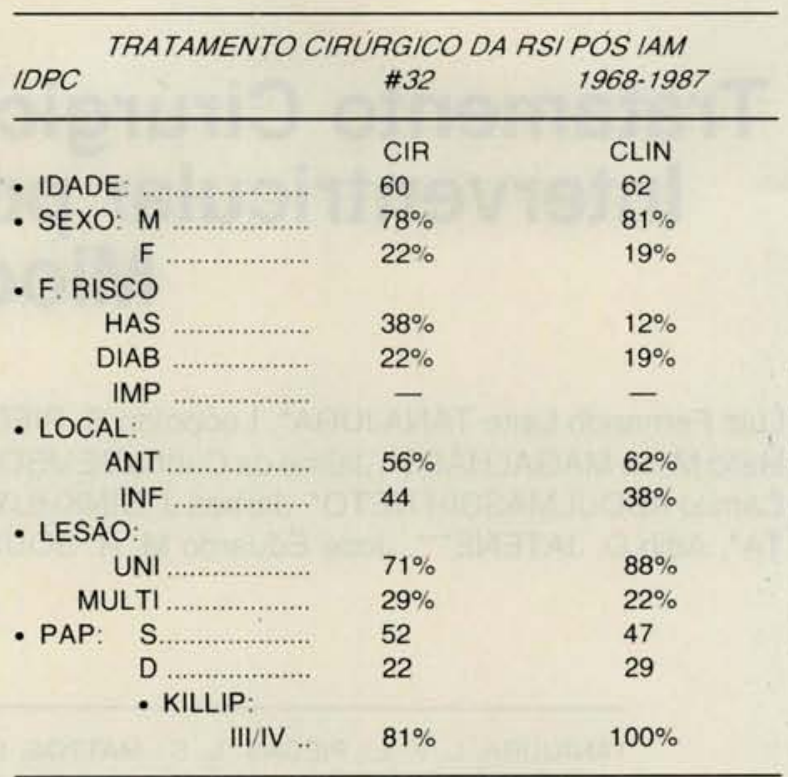

variando de 47 a 77 anos (média de 60 anos). Tinham antecedentes de hipertensão arterial $12(38 \%)$ e diabetes $7(22 \%)$; nenhum paciente apresentava infarto prévio.

O diagnóstico do IAM foi feito através dos critérios clássicos: clínico, eletrocardiográfico e enzimático. Estava localizado na parede anterior em $18(56 \%)$ e na parede inferior em $14(44 \%)$.

O surgimento do sopro sugestivo da complicação, naqueles casos em que foi possível estabelecer uma data precisa, ocorreu na primeira semana em $9(47 \%)$ e na segunda semana em $10(53 \%)$.

Disfunção ventricular esquerda severa, graus III e IV da classificação de Killip, foi encontrada em 26 (81\%) grau II em $5(16 \%)$ e grau I em $1(3 \%)$. Arritmias graves (taquicardia paroxística supraventricular, fibrilação e flutter atrial, taquicardia ventricular, fibrilação ventricular e bloqueios de diferentes graus) foram encontradas em $13(41 \%)$.

O diagnóstico clínico de RSI foi comprovado através de estudo hemodinâmico à beira do leito obtido com o catéter de Swan-Ganz em 18 (56\%) e/ou cineangiocoronariografia em $31(97 \%)$. As pressōes de artéria pulmonar mostraram-se sistematicamente elevadas ${ }^{17}$ (Gráfico 1).

O débito cardiaco, aferido pela técnica da termodiluição, situou-se em níveis "normais", na grande maioria dos casos, o que se deve ao erro introduzido neste cálcu-

- OYAMADA, A. \& QUEEN, F. B. - Spontaneous rupture of the interventricular septum following acute myocardial infarction with some clinicopathological observations on survival in five cases. Apresentado ao Pan Pacific Pathology Congress. Triple U.S.A. Army Hospital, 1961 
TANAJURA. L. F. L.: PIEGAS. L. S.: MATTOS. L. A.: PINTO. I. F: MAGALHÄES. H. M. BEMBOM. J C ARNONI. A S. OLIVEIRA. J. B.: ABDULMASSIH NETO. C.: DINKHUYSEN. J. J.: SOUZA. L. C. B.: PAULISTA. P. P.: JATENE. A. D.: SOUSA J. E. M. R. - Tratamento cirurgico da rotura do septo interventricular pos infarto agudo do miocardio. Rev. Bras. Cir Cardiovasc. 2(2): 87-92. 1987

lo pelo shunt em nivel ventricular; a magnitude deste, avaliada através de oximetria, mostrou ser importante (Gráfico 2).

\section{GRÁFICOS 1 e 2}

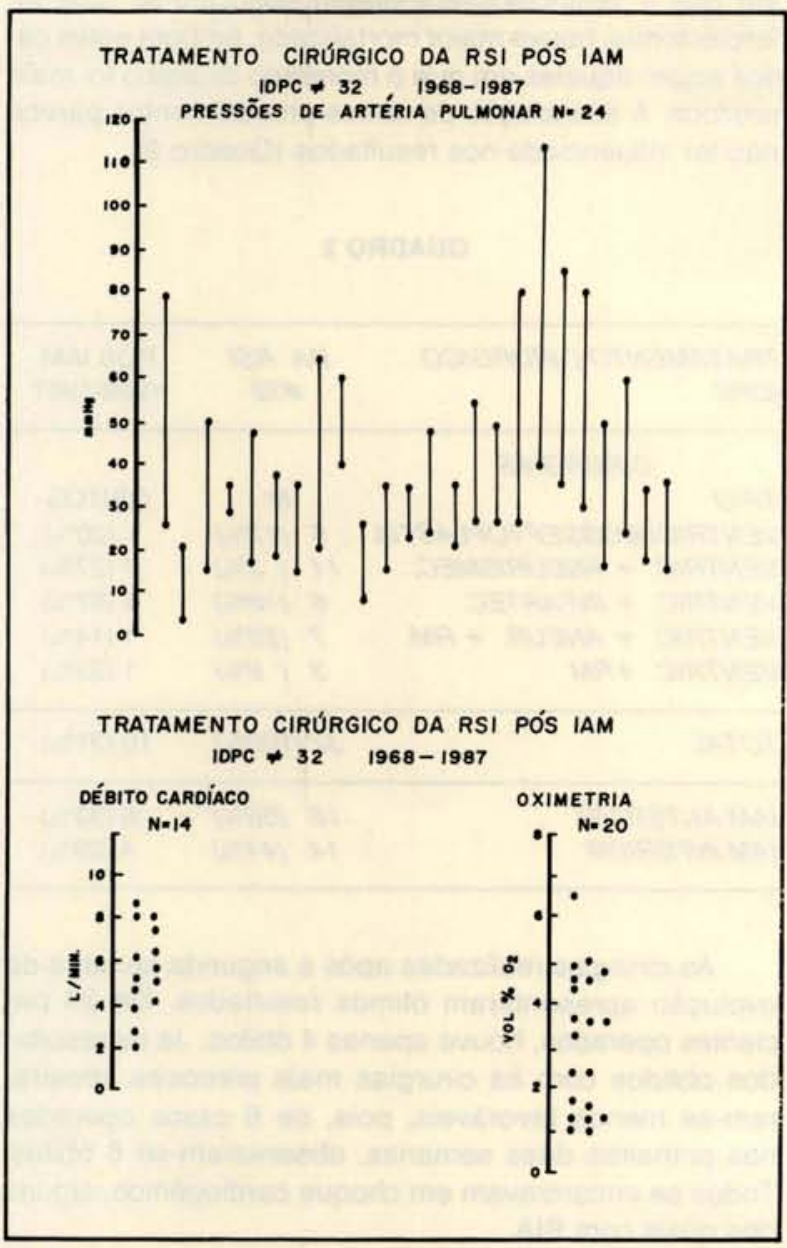

Todos os pacientes que realizaram cinecoronariografia apresentavam lesōes coronárias severas (obstrução igual ou superior a $70 \%)$, sendo $22(71 \%)$ uniarteriais e $9(29 \%)$ multiarteriais, todos com lesões de dois vasos. Dentre os que apresentavam lesão em um único vaso, a descendente anterior estava comprometida em 15 (68 $\%)$, a coronária direita em $6(27 \%)$ e a artéria circunflexa em $1(5 \%)$. Aneurismas ventriculares associados foram encontrados em $22(71 \%)$.

O tratamento cirúrgico foi realizado nas duas primeiras semanas de evolução em 8 pacientes (25\%), e, após esse período, em 24 (75\%), com mortalidades respectivas de $6(75 \%)$ e $4(17 \%)$. A mortalidade global foi de 10 (31\%). Em 7 (22\%), foi usado o BIA no pós-operatório, sendo que, em $4(13 \%)$, já fora introduzido no pré-operatório. Destes, faleceram 5 (71\%).

As causas de óbito no pós-operatório foram choque cardiogênico em $8(80 \%)$, acidente vascular cerebral em
$1(10 \%)$ e rotura de VE em $1(10 \%)$, tendo, como causas associadas, insuficiência renal aguda em 4 , hemorragia digestiva em 2 e recidiva da perfuração ainda na sala de cirurgia em 1 . Em relação à topografia do infarto, dos 10 óbitos cirúrgicos, $6 / 18(33 \%)$ eram infartos anteriores e $4 / 14(29 \%)$, inferiores.

Dos 22 (69\%) sobreviventes, $14(64 \%)$ encontramse assintomáticos, $1(5 \%)$ discretamente sintomático (dispnéia aos grandes esforços); ocorreram 4 (18\%) óbitos ( 3 por insuficiência cardiaca e 1 de causa ignorada) e perdeu-se a evoluçāo de $3(13 \%)$.

Reestudaram-se através da cinecoronariografia, 9 (41\%) pacientes, observando-se manutenção do resultado cirúrgico em $7(78 \%)$, recidiva da complicação em $1(11 \%)$ (o qual foi posteriormente operado) e discreto shunt residual em $1(11 \%)$. Comprovaram-se melhora da contratilidade ventricular, normalização das pressōes na artéria pulmonar e patência das pontes de safena naqueles que as receberam.

\section{DISCUSSÃO}

Nos últimos 19 anos, 32 pacientes foram internados no IDPC, e operados com diagnóstico de RSI pós IAM. Neste grupo, nāo houve predominância de sexo, grupo etário, ou fatores de risco, estando estas variáveis correlacionadas com o infarto e não com a complicação. Curiosamente, nenhum deles apresentava infarto prévio, suspeito, ou confirmado.

A incidência desta complicação, em nossa casuística, situa-se em torno de $2,5 \%$, semelhante à registrada na literatura ${ }^{3,11,15}$.

Houve um predomínio discreto dos infartos que acometeram a parede anterior (18/32), embora sem significado estatístico. Os padrōes eletrocardiográficos foram bem claros, não deixando dúvidas quanto ao diagnóstico do IAM. A época de surgimento do sopro também foi semelhante à relatada na literatura ${ }^{11}$.

As manifestações clínicas e propedêuticas da RSI, bem como seu aspecto evolutivo, comumente confundem-se com as da regurgitação mitral ocasionada pela disfunção ou rotura de músculo papilar, só podendo ser corretamente diferenciadas através dos parâmetros hemodinâmicos. Estes são facilmente obtidos com estudo hemodinâmico à beira de leito, realizado com catéter de Swan-Ganz. Esta técnica, sempre que utilizada, proporcinou a confirmação do diagnóstico dentro da unidade coronária, bem como uma abordagem terapêutica mais adequada (PIEGAS et alii ${ }^{17,{ }^{18}}$ ).

Uma das características da complicação é o surgimento de manifestações severas de falência ventricular esquerda, geradas pelo desarranjo hemodinâmico oca- 
TANAJURA. L. F. L. PIEGAS. L. S: MATTOS. L. A.: PINTO. I. F: MAGALHÄES. H M. BEMBOM. J. C.: ARNONI. A. S OLIVEIRA. J. B.: ABDULMASSIH NETO. C.: DINKHUYSEN. J. J.: SOUZA. L. C B.: PAULISTA. P. P.: JATENE. A. D.: SOUSA. J. E. M. R. - Tratamento cirúrgico da rotura do septo interventricular pos infarto agudo do miocardio. Rev. Bras. Cir. Cardiovasc., 2(2): 87-92, 1987

sionado pelo shunt ventricular. Nesta casuística, 26 ( $81 \%)$ pertenciam aos grupos III e IV da classificação de Killip (KITAMURA et alii ${ }^{9}$ e PIEGAS et alii ${ }^{18}$ ).

A presença de hipertensão arterial pulmonar também é uma constante, nestes casos, chegando, por vezes, a igualar os níveis sistêmicos (Gráfico 1). É gerada, em parte, pelo componente hipercinético, que decorre do hiperfluxo pulmonar secundário à comunicação interventricular (o que é confirmado pelos importantes saltos oximétricos observados e pela regressão das alteraçōes pressóricas após sua correção), podendo, também, ser devida à diminuição da complacência ventricular secundária à necrose miocárdica (PIEGAS et alii ${ }^{18}$ ).

Sempre que possivel, indicou-se a cinecoronariografia precocemente, não se aguardando mais que 24 horas para tentar estabilizar as condiçōes hemodinâmicas adversas. Muitos foram levados à sala de exames em choque cardiogênico, sem que houvesse agravamento de sua condição clínica.

Nesta experiência, houve um predomínio de casos com lesōes uniarteriais, já que, dos 31 pacientes estudados, apenas $9(29 \%)$ apresentavam comprometimento severo de dois vasos. Nos portadores de doença uniarterial, o vaso mais freqüentemente acometido foi a descendente anterior ( 15 casos), seguindo-se a coronária direita ( 6 casos) e a artéria circunflexa (1 caso). O fato de encontrarmos apenas 1 caso isolado, de acometimento da circunflexa, é explicado pela raridade com que esta artéria contribui para a irrigação do septo interventricular.

Outro achado constante na cinecoronariografia foi a presença de aneurismas ventriculares, cujo aparecimento se deve à ocorrência de extensa necrose miocárdica. Foram observados em $22(71 \%)$ dos pacientes estudados.

Devido às importantes alteraçōes anatomofuncionais secundárias à RSI, o único método terapêutico efetivo para controlar as manifestaçōes de falência miocárdica é a cirurgia corretiva. O momento adequado para sua realização pōe o cardiologista frente a um dilema: se for precoce, poderá se defrontar com uma maior mortalidade operatória; se protelada, certamente haverá, também, um elevado número de óbitos, devido à impossibilidade de estabilização clínica das condiçōes hemodinâmicas, mesmo valendo-se dos mais recentes esquemas terapêuticos (vasodilatadores, inotrópicos e BIA).

Como se frisou no início deste trabalho, a totalidade dos pacientes mantidos clinicamente faleceu, quer por se estar aguardando um melhor momento para a intervenção cirúrgica, quer pela impossibilidade de desencadeá-la. Analisando-se a época em que ocorreram os óbitos, notou-se que $81 \%$ deles se deram nas duas primeiras semanas de evolução, o que parece ser um dado a favor da intervenção precoce ${ }^{12,18}$.
Operaram-se 32 pacientes, com mortalidade de 10 $(31 \%)$. As cirurgias e os respectivos óbitos estāo relacionados no Gráfico 2. Estes dados não são expressivos, para que se possa comparar os diversos procedimentos e sua mortalidade. Apenas parece que, naqueles casos em que à ventriculosseptoplastia associou-se uma infarctectomia, houve maior mortalidade, embora estes casos sejam aqueles em que o momento cirúrgico foi mais precoce. A associação de outros procedimentos parece não ter influenciado nos resultados (Quadro 2).

\section{QUADRO 2}

\begin{tabular}{lcc}
\hline $\begin{array}{l}\text { TRATAMENTO CIRÚRGICO } \\
\text { IDPC }\end{array}$ & $\begin{array}{c}\text { DA RSI } \\
\# 32\end{array}$ & $\begin{array}{c}\text { PÓS IAM } \\
1968-1987\end{array}$ \\
\hline \multicolumn{1}{c}{ CIRURGIAS } & & \\
TIPO & $N$. & OBITOS \\
VENTRICULOSSEPTOPLASTIA & $5(17 \%)$ & $1(20 \%)$ \\
VENTRIC. + ANEURISMEC. & $11(3 \%)$ & $3(27 \%)$ \\
VENTRIC. + INFARTEC. & $6(18 \%)$ & $4(67 \%)$ \\
VENTRIC. + ANEUR. + RM & $7(22 \%)$ & $1(14 \%)$ \\
VENTRIC. +RM & $3(9 \%)$ & $1(33 \%)$ \\
\hline TOTAL & $32(100 \%)$ & $10(31 \%)$ \\
\hline IAM ANTERIOR & $18(56 \%)$ & $6(33 \%)$ \\
IAM INFERIOR & $14(44 \%)$ & $4(29 \%)$ \\
\hline
\end{tabular}

As cirurgias realizadas após a segunda semana de evolução apresentaram ótimos resultados. De 24 pacientes operados, houve apenas 4 óbitos. Já os resultados obtidos com as cirurgias mais precoces, mostraram-se menos favoráveis, pois, de 8 casos operados nas primeiras duas semanas, observaram-se 6 óbitos. Todos se encontravam em choque cardiogênico, alguns dos quais com BIA.

A localização do infarto, condicionando o local da rotura do septo (anterior nos infartos anteriores e póstero-inferior nos infartos inferiores) não influiu nos resultados. Dos 18 pacientes operados que apresentavam necrose anterior, faleceram $6(33 \%)$, enquanto que, dos 14 pacientes cirúrgicos com infarto inferior, morreram $4(29 \%)$.

Apesar dos resultados obtidos com a cirurgia precoce (duas primeiras semanas) terem sido pouco animadores nesta experiência, acreditamos que a indicação cirúrgica não deve ser protelada. Vários grupos já obtiveram bons resultados, nesta fase, o que se deve, provavelmente, ao aprimoramento das técnicas cirúrgicas e emprego da cardioplegia. Além disto, deve-se ter em mente que estes doentes têm poucas possibilidades de sobreviver sem uma correção cirúrgica ${ }^{5}, 6,8,12,13,18,19,22$.

A evoluçāo tardia apresentada pelos sobreviventes cirúrgicos tem-se mostrado extremamente favorável. 
TANAJURA, L. F. L.: PIEGAS, L. S.: MATTOS, L. A: PINTO. I. F: MAGALHÄES, H. M: BEMBOM. J. C.: ARNONI. A. S. OLIVEIRA, J. B.: ABDULMASSIH NETO, C.: DINKHUYSEN. J. J.: SOUZA. L. C. B.: PAULISTA. P. P.: JATENE. A. D : SOUSA. J. E. M. R. - Tratamento cirúrgico da rotura do septo interventricular pos infarto agudo do miocardio. Rev. Bras. Cir. Cardiovasc. 2(2): 87-92, 1987.

Dos 22 pacientes que tiveram alta hospitalar, $14(64 \%)$ encontram-se assintomáticos, $1(5 \%)$ tem sintomatologia discreta, $4(18 \%)$ faleceram durante a internação (dos quais 3 por insuficiência cardiaca) e perdeu-se a evolução de $3(13 \%)$ pacientes.

A recidiva do defeito foi infreqüente. De 9 resultados, observou-se apenas $1(11 \%)$ que apresentava recidiva significativa (foi, curiosamente, o primeiro da série); outro paciente apresentava discreto shunt residual sem significado hemodinâmico, sendo mantido em evolução clínica.
Concluindo, frente à rápida deterioração hemodinâmica apresentada pelos pacientes com RSI pós IAM, quando mantidos em tratamento clínico, parece ser mais correto encaminhá-los precocemente à correção cirúrgica. Embora a sobrevida seja maior quanto mais tardiamente se opere, os doentes que superam a fase aguda apenas com tratamento clínico parecem constituir um grupo selecionado e de melhor prognóstico, diferindo da evoluçāo tormentosa que, habitualmente, costumam exibir os portadores desta complicação.

\section{RBCCV $44205-26$}

TANAJURA, L. F. L.; PIEGAS, L. S.; MATTOS, L. A.; PINTO, I. F.; MAGALHÃES, H. M.; BEMBOM, J. C.; ARNONI, A. S.; OLIVEIRA, J. B.; ABDULMASSIH NETO, C.; DINKHUYSEN, J. J.; SOUZA, L. C. B.; PAULISTA, P. P.; JATENE, A. D.; SOUSA, J. E. M. R. - Surgical treatment of ventricular septal defect post acute myocardial infart. Rev. Bras. Cir. Cardiovasc., 2(2): 87-92, 1987.

ABSTRCT: The ventricular septal rupture (VSR) following acute myocardial infarction (AMI) is a rare and catasthrophic complication with a high mortality rate. Surgery is the only effective procedure to try to control this complication. However the surgical mortality is elevated and is closely related to the post-infarct period thus making the indication for the precise time for surgery one the fundamental aspects for its success. In a population of 48 patients with diagnosis of VSD following AMI, according to the criteria of the Myocardial Infarction Research United, we treated $32(67 \%)$ with surgery. The diagnosis was confirmed by hemodynamic in all patients. The AMI was anterior in 18 cases $(56 \%)$ and inferior in $14(44 \%)$; the mortality rate is $6(33 \%)$ and $4(29 \%)$ cases respectively. Severe heart failure (Killip III and IV) was present in 26 cases $(81 \%)$ and life threatening arrhythmias in $13(41 \%)$. A Swan-Ganz catheter was introduced at bedside in $18(56 \%)$ patients to confirm the diagnosis and provide a better therapeutic management. Angiographic studies were performed in $31(97 \%)$. Cineangiography showed a critical lesion (obstruction equal or greater than $70 \%$ of the lumen) in one vessel in 22 patients $(71 \%)$ : left anterior descending coronary artery in $15(68 \%)$, right coronary artery in $6(27 \%)$ and left circunflex in $1(5 \%)$. Nine cases $(29 \%)$ had critical lesions in two vessels. The pulmonary artery pressure was always elevated in those patients in whom the pressure was measured. Clinical treatment was used in $16(33 \%)$ cases and all died during hospitalization. Eight patients $(25 \%)$ underwent the surgery within the first two weeks and $24(75 \%)$ after this period; mortality rate was $6(75 \%)$ and $4(17 \%)$ cases respectively. Nine patients were re-catheterized in the post operative period and only one presented signs of significant shunt in the ventricular level. This patient who was the oldest of the group was reoperated later. Of the 22 patients discharged from the hospital 15 are long-term survivors. In 3 the follow-up was lost and 4 died. The accumulated data allows us to conclude that: 1) the attempt to postpone surgery by keeping the patients under medical treatment waiting for a more favorable time for surgery did not produce satisfactory results; 2) surgery should be performed in the acute phase (first two weeks) in spite of a still elevated mortality in this group; 3 ) after the first two weeks the results are good with low mortality rate for this kind of surgery; 4) VSD reappearence was infrequent.

DESCRIPTORS: ventricular septal defect, surgery; myocardial infarct, surgery.

\section{REFERÊNCIAS BIBLIOGRÁFICAS}

1 BRUNN, F. - Zur Diagnostic der erworbenen Ruptur der Kammersscheidewand des Herzens, Wien. Arch. Int. Med., 6: 533-544, 1923.

2 BURAKOVSKY, V. I.; TSUKERMAN, G. I.; KRAKOVSKY, A. A. - Basic courses of approach to the surgical treatment of postinfarct defects of the interventricular septum. Kardiologiia, 19 (1): 8-15, 1979.

3 CAMPION, B. C.; HARRISON, C. E.; GUILIANI, E. R. Ventricular septal defect after myocardial infarction. Ann. Int. Med., 70 (2): 251-261, 1969.
4 COOLEY, D. A.; BELMONTE, B. A.; ZEIS, L. B.; SCHNUR, S. - Surgical repair of ruptured interventricular septum following acute myocardial infarction. Surgery, 41: 930-937, 1957

5 DAGGETT, W. M.; GUYTON, R. A.; MUNDTH, E. D.; BUCKLEY, M. J.; MCENANY, M. T.; GOLD, H. K.; LEINBACH, R. C.; AUSTEN, W. G. - Surgery for post-myocardial infarct ventricular septal defect. Ann. Surg., 186 (3): 268-271, 1977.

6 HILL, J. D.; LARY, D.; KERTH, W. J.; GERBODE, F. Acquired ventricular septal defects. J. Thorac. Cardiovasc. Surg., 70 (3): 440-450, 1975. 
TANAJURA, L. F. L.: PIEGAS. L. S: MATTOS. L. A: PINTO. I. F: MAGALHẢES. H. M.: BEMBOM. J. C: ARNONI. A. S. OLIVEIRA. J. B : ABDULMASSIH NETO. C.: DINKHUYSEN. J. J.: SOUZA. L. C. B.: PAULISTA. P. P . JATENE. A. D.: SOUSA. J. E. M. R. - Tratamento cirurgico da rotura do septo interventricular pos infarto agudo do miocardio. Rev. Bras. Cir. Cardiovasc., 2(2): 87-92. 1987.

7 HUTCHINS, G. M. - Rupture of the interventricular septum complicating myocardial infarction: pathological analysis of 10 patients with clinically diagnosed perforations. Am. Heart J., 97 (2): 165-173, 1979.

8 IBEN, A. B.; PUPELLO, D. F.; STINSON, E. B.; SHUMWAY, N. E. - Surgical treatment of postinfarction ventricular septal defects. Ann. Thorac. Surg., 8 (3): 252-262, 1969.

9 KITAMURA, S.; MENDEZ, A.; KAY, J. H. - Ventricular septal defects following myocardial infarction. J. Thorac. Cardiovasc. Surg., 61 (2): 186-199, 1971.

10 LATHAM, P. M. - Lectures on subjects connected with clinical medicine compromising disease of the heart. London, Brown, Green and Longmans, 1945. p. 163-164.

11 LEE, W. Y.; CARDON, L.; SLODKI, S. J. - Perforation of infarcted interventricular septum. Arch. Int. Med., 109 (6): 731-741, 1962.

12 LEMERY, R.; SMITH, H. C.; GERSH, B. J.; GIULIANI, E. R.; SCHAFF, H. V. - Prognosis of ventricular septal defect following acute myocardial infarction. J. Am. Coll. Cardiol., 7(2): 33, 1986.

13 LOISANCE, D. Y.; CACHERA, J. P.; POULAIN, H.; AUBRY, P.; JUVIN, A. M.; GALEY, J. J. - Ventricular septal defect after acute myocardial infarction. J. Thorac. Cardiovasc. Surg., 80: (1): 61-67, 1980.

14 MONTOYA, A.; MCKEEVER, L.; SCANLON, P.; SULLIVAN, H. J.; GUNNAR, R. M.; PIFARRE, R. - Early repair of ventricular septal rupture after infarction. $A m$. J. Cardiol., 45 (2): 345-348, 1980.

15 OLDHAM Jr. H. N.; SCOTT, S. M.; DART Jr., C. H. Surgical correction of ventricular septal defect following acute myocardial infarction. Ann. Thorac. Surg., 7 (3): 193-201, 1969.
16 PETROV, K.: KARALAMBEV, N.: TONEVA, N.: VASSILEV, C. - Analyse de 153 cas de rupture du myocarde apres l'infarctus aigu du myocarde. Arch. Mal. Coeur. 70 (7): 691-697, 1977.

17 PIEGAS, L. S.: CARVALHO, H. G.: SOUSA, J. E. M. R. RESECK, P. A. R.: JATENE, A. D. - Use of the SwanGanz catheter in the diagnosis of ventricular septal defect after myocardial infarction: case report. Heart Lung. 2 (4): 539-541, 1973.

18 PIEGAS, L. S.: SANTOS, R. J.: MIRANDA, M. A. T.: BEMBOM, J. C.; TIMERMAN, A.; BARBOSA, M. A. O.; EGITO, E. T.: ABDULMASSIH NETO, C.; SOUZA, L. C. B.; PAULISTA, P. P.; JATENE, A. D. - Ruptura do septo interventricular pós infarto agudo do miocádio: o dilema da indicaçâo cirúrgica. Arq. Bras. Cardiol. 37 (4): 241-246, 1981.

19 PIWNICA, A.; NOTTIN, R.; BERCOT, M.; DELOCHE, A.: D'ALLAINES, C.; CARPENTIER, A.; BLONDEAU, P.: DUBOST, C. - Resultats du traitement chirurgical de 31 cas de perforation septale operés au cours des 21 premier jours de l'infarctus myocardique. Ann. Chir. Thorac. Cardiovasc., 30 (9): 257-262, 1976.

20 SAGER, R. V. - Coronary thrombosis: perforation of the infarcted interventricular septum. Arch. Int. Med., 53 (1): $140-148,1934$.

21 SANDERS, R. J.; KERN, W. H.; BLOUNT Jr., S. G. Perforation of the interventricular septum complicating myocardial infarction: a report of eight cases, one with cardiac catheterization. Am. Heart J., 51 (5): 736-748. 1956.

22 STINSON, E. B.; BECKER, R. J.; SHUMWAY, N. E. Successful repair of post infarction ventricular septal defect by biventricular aneurysm. J. Thorac. Cardiovasc. Surg., 58 (1): 20-24, 1969

23 VLODAVER, Z. \& EDWARDS, J. E. - Rupture of ventricular septum or papillary muscle complicating myocardial infarction. Circulation, 55 (5): 815-822, 1977. 